\title{
Awareness and Involvement of the Arts, Commerce and Science College Teachers in Students' Mental Health and Behavioural Problems: A Cross-sectional Study from Sangli District $(\mathrm{MH})$, India
}

\author{
Vivek Baliram Waghachavare, ${ }^{a}$ Manohar Shankarrao Chavan, ${ }^{b}$ Alka Dilip Gore, ${ }^{a}$ Girish Bhimrao Dhumale ${ }^{a}$ \\ ${ }^{a}$ Department of Community Medicine, Bharati Vidyapeeth Deemed University Medical College \& Hospital, \\ Sangli (Maharashtra) India \\ ${ }^{b}$ Department of Medicine, Bharati Vidyapeeth Deemed University Medical College \& Hospital, Sangli \\ (Maharashtra) India - 416414.
}

\begin{abstract}
Background: Teachers play an important role in reducing adolescent health problems. However, the involvement of teachers in adolescent health is not well understood. This current study was conducted to address this. Methods: A cross-sectional study was conducted among teachers of randomly selected arts, commerce and science colleges in Sangli, (Maharashtra) India. The data was collected by cluster random sampling. The study period was from November 2014 to April 2015. The study tool was self-administered questionnaire. Due ethical considerations, consents were taken. Analysis was done using Microsoft Excel 2003 and IBM SPSS version 22. Results: Out of 319 study participants; 246 (77.1\%) participants had good awareness regarding adolescent health problems, 254 (79.6\%) participants had good attitude towards reproductive health. Forty-three (13.5\%), 22 (6.9\%) and 15 (4.7\%) participants had good practices regarding behavioural problems, psychological problems and substance abuse respectively. Practices about behavioural problems and substance abuse were associated with adolescent health training. In bi-variate analysis, age was a significant predictor for all the three practices. Conclusion: Most teachers had good attitude and awareness; but poor knowledge and practices regarding adolescent issues. All teachers should undergo training regarding adolescent health; with periodic refresher courses or lectures. Anonymous feedback from students should be sought to keep vigilance over efforts of the teachers.
\end{abstract}

Keywords: Adolescent, Adolescent Psychology, Behaviour, Faculty.

\section{INTRODUCTION}

World Health Organisation (WHO) defines adolescence phase from 10 to 19 years of age. ${ }^{1}$ The onset of puberty has long been accepted as the starting point of adolescence, and key social-role transitions such as completion of education, employment, marriage, and childrearing historically signalled the end. ${ }^{2}$ Puberty triggers emotional, cognitive, and behavioural changes which may result in increased mortality and morbidity from accidental and intentional injuries, suicide and mental disorders, substance abuse, and eating disorders in young people. ${ }^{3}$ Out of the estimated

Corresponding author:

Vivek Baliram Waghachavare

Department of Community Medicine,

Bharati Vidyapeeth Deemed University Medical

College \& Hospital,

Sangli (Maharashtra)

India - 416414.

Email: vivek416416@gmail.com global adolescent population of 1.2 billion, India is largest contributor with 243 million adolescents. ${ }^{4,5}$ This impresses the need of proper nurturing of adolescents.

Adolescent health is a much younger discipline by comparison to Paediatrics. The problem of deficit in specialized medical care and due attention of policy makers regarding adolescent health is further escaladed due to lack of health seeking behaviour; especially mental health. ${ }^{6,7}$ The problem is acute in developing countries, where even adult mental health-seeking is poor. ${ }^{8,9}$

Teachers can play an important role as a source of knowledge, counsellor and aid in developing proper attitude among adolescents. It has been said that strong student-teacher engagement results in reduced substance abuse, and behavioural problems. ${ }^{2}$ They can also help in early suspicion of a health problem and referral. But teachers need to know the signs and symptoms about various adolescent problems, so that they can help students. $^{4}$ 
But the research as well as efforts regarding incorporating active involvement of teachers in the field of adolescent health is negligible and so is the knowledge of teachers regarding adolescent health. ${ }^{10}$ The situation worsens in the colleges. This study was planned to look into awareness and involvement in the adolescent health of the graduation college teachers belonging to the Sangli district, Maharashtra.

\section{METHODS}

This is a cross-sectional study. The study area was arts, commerce and science degree (graduation) colleges from the Sangli District, (Maharashtra) India; with reference population of teachers from these colleges. Sample size based on results of pilot study was 265 ( $p$ of awareness $=79 \%$, error $5 \%$ ). The study period was six months, from November 2014 to April 2015.

For the study, twelve arts, commerce, science degree (graduation) colleges were randomly selected out of total pool of 52 colleges. The data was collected by cluster random sampling technique. Teachers who refused consent or those who were not comfortable with English questionnaire were excluded from the study.

After the study proposal was scrutinized and approved by the Scientific Research Review committee and Institutional Ethical Committee; written permission was acquired from the head of the selected institutes. Written informed consent was acquired from every participant.

The study tools were self-administered. Pre-tested questionnaire based on published literature was developed with the help of specialists from the field and experienced faculty. ${ }^{11-14}$ A pilot study was conducted, and the questionnaire was modified and finalized based on the pilot study.

The questionnaire had eight sections based on the outcome expected. Section 1 consisted of sociodemographic variables along with information regarding their experience and training in adolescent health. Section 2 had a list of various psychological, behavioural and other health problems and participants were requested to identify which were commonly present in the adolescents. This section was developed to assess the awareness of the teachers regarding adolescent health problems. Section 3 was regarding the attitude of teachers towards adolescent reproductive health. Section 4 had questions regarding symptoms of various mental health problems based on DSM-IV criteria, to assess the knowledge about psychological problems. ${ }^{11}$

This section, being knowledge score was examined for reliability. It had good internal consistency (Cronbach's a, $r=0.72$ ). Section 5 had questions regarding identification of signs of various substance abuse. Section 6, 7 and 8 were regarding practices undertaken by the teachers if they identify students with psychological, substance abuse and behavioural problem respectively.

For section 2 to 8 , desirable or correct answers were predefined. All correct answers were given a score of 1 and undesirable or wrong answers were given a score of 0 . Total score for each section was calculated separately. After consultation with field experts, it was decided to consider the score of $\leq$ $50 \%$ as "poor" score in that particular section and score of > 50\% to be "good" score. All subsections were individually examined for test-retest reliability and they were satisfactorily reliable.

After the requisite permissions, teachers were contacted in the staff room. After ensuring voluntary participation, informed consent, adequate privacy and appropriate pre-counselling; questionnaires were given to them. All questionnaires were collected in a drop-box. Incomplete questionnaires were not included in the final analysis.

Analysis was done using Microsoft Excel 2003 and IBM SPSS-22. Percentage, chi-square test and regression were used in the analysis. Data from the pilot study was not included in the final analysis.

\section{RESULTS}

Out of the 347 questionnaires collected from the study participants, 319 were completely filled and hence included in the analysis. One hundred and thirteen i.e. $35.4 \%$ participants were females and $206(64.6 \%)$ were males. The youngest participants were at the age of 23 years while the eldest were of 60 years. The mean age of participants was 40.5 years (standard deviation 10.6 years); and median of age was 40 years. (Figure 1)

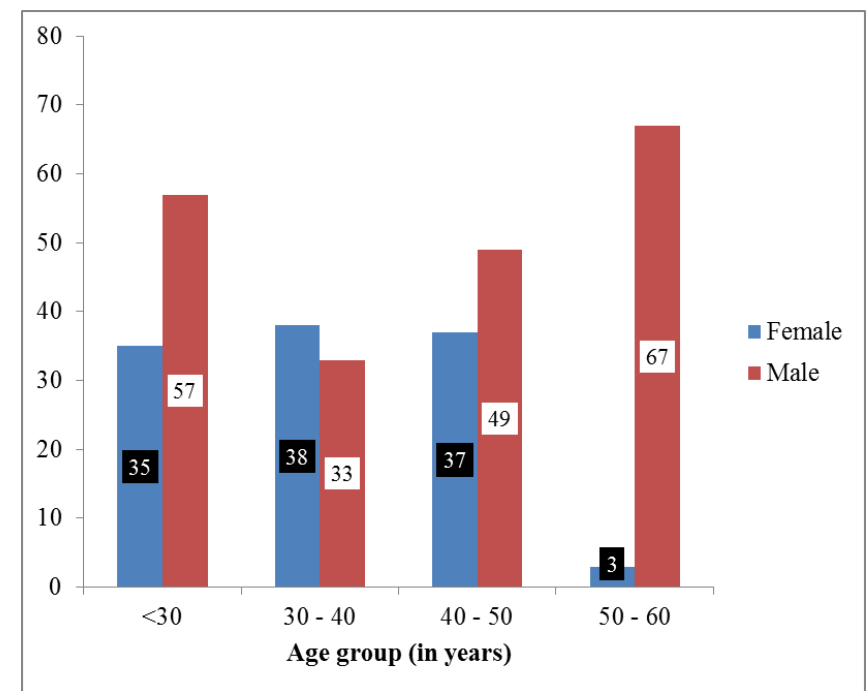

Figure 1: Age and sex distribution of teachers

Considering the designation of the study participants, 197 (61.8\%) were tutors or lecturers, 122 (38.2\%) were associate professors or professors. 
The mean experience of teaching was 15.1 years (standard deviation 10.1 years). On dividing the participants in different teaching experience groups, $78(24.5 \%)$ and 241 (75.5\%) belonged to groups of $\leq 5$ years and $>$ 5years respectively. Only $90(28.2 \%)$ participants had undergone some training or workshop regarding adolescent health; all of them had received such training only once.

Table I: Association of awareness regarding adolescent health problems and attitude towards reproductive health with various socio-demographic variables

\begin{tabular}{|c|c|c|c|c|c|c|}
\hline \multicolumn{2}{|l|}{ Variables } & \multicolumn{2}{|c|}{$\begin{array}{l}\text { Awareness regarding adolescent } \\
\text { health problems }\end{array}$} & \multicolumn{2}{|c|}{$\begin{array}{l}\text { Attitude towards reproduc- } \\
\text { tive health }\end{array}$} & \multirow{3}{*}{$\begin{array}{l}\text { Total } \\
163\end{array}$} \\
\hline & & Poor & Good & Poor & Good & \\
\hline \multirow{5}{*}{$\begin{array}{l}\text { Age group } \\
\text { (in years) }\end{array}$} & \multirow{2}{*}{$\leq 40$} & 31 & 132 & 29 & 134 & \\
\hline & & $19 \%$ & $81 \%$ & $17.8 \%$ & $82.2 \%$ & $100 \%$ \\
\hline & \multirow{2}{*}{$>40$} & 42 & 114 & 36 & 120 & 156 \\
\hline & & $26.9 \%$ & $73.1 \%$ & $23.1 \%$ & $76.9 \%$ & $100 \%$ \\
\hline & \multicolumn{3}{|c|}{ Chi-square $=2.822, p=0.093$} & \multicolumn{3}{|c|}{ Chi-square $=1.373, p=0.241$} \\
\hline \multirow{5}{*}{ Gender } & \multirow{2}{*}{ Female } & 15 & 98 & 26 & 87 & 113 \\
\hline & & $13.3 \%$ & $86.7 \%$ & $23.0 \%$ & $77.0 \%$ & $100 \%$ \\
\hline & \multirow{2}{*}{ Male } & 58 & 148 & 39 & 167 & 206 \\
\hline & & $28.2 \%$ & $71.8 \%$ & $18.9 \%$ & $81.1 \%$ & $100 \%$ \\
\hline & \multicolumn{3}{|c|}{ Chi-square $=9.157, p=0.002$} & \multicolumn{3}{|c|}{ Chi-square $=0.748, p=0.387$} \\
\hline \multirow{5}{*}{ Designation } & \multirow{2}{*}{$\begin{array}{l}\text { Tutor/ } \\
\text { Lecturer }\end{array}$} & 41 & 156 & 35 & 162 & 197 \\
\hline & & $20.8 \%$ & $79.2 \%$ & $17.8 \%$ & $82.2 \%$ & $100 \%$ \\
\hline & \multirow{2}{*}{$\begin{array}{l}\text { Associate } \\
\text { Professor / } \\
\text { Professor }\end{array}$} & 32 & 90 & 30 & 92 & 122 \\
\hline & & $26.2 \%$ & $73.8 \%$ & $24.6 \%$ & $75.4 \%$ & $100 \%$ \\
\hline & \multicolumn{3}{|c|}{ Chi-square $=1.253, p=0.263$} & \multicolumn{3}{|c|}{ Chi-square $=2.162, \mathrm{p}=0.141$} \\
\hline \multirow{5}{*}{$\begin{array}{l}\text { Adolescent } \\
\text { Health } \\
\text { training }\end{array}$} & \multirow{2}{*}{ No } & 53 & 176 & 50 & 179 & 229 \\
\hline & & $23.1 \%$ & $76.9 \%$ & $21.8 \%$ & $78.2 \%$ & $100 \%$ \\
\hline & \multirow{2}{*}{ Yes } & 20 & 70 & 15 & 75 & 90 \\
\hline & & $22.2 \%$ & $77.8 \%$ & $16.7 \%$ & $83.3 \%$ & $100 \%$ \\
\hline & \multicolumn{3}{|c|}{ Chi-square $=0.031, p=0.86$} & \multicolumn{3}{|c|}{ Chi-square $=1.063, \mathrm{p}=0.302$} \\
\hline \multirow{5}{*}{ Experience } & \multirow{2}{*}{$\leq 5$ yrs } & 14 & 64 & 11 & 67 & 78 \\
\hline & & $17.9 \%$ & $82.1 \%$ & $14.1 \%$ & $85.9 \%$ & $100 \%$ \\
\hline & \multirow{2}{*}{$>5$ yrs } & 59 & 182 & 54 & 187 & 241 \\
\hline & & $24.5 \%$ & $75.5 \%$ & $22.4 \%$ & $77.6 \%$ & $100 \%$ \\
\hline & \multicolumn{3}{|c|}{ Chi-square $=1.425, p=0.233$} & \multicolumn{3}{|c|}{ Chi-square $=2.505, p=0.114$} \\
\hline \multirow{2}{*}{\multicolumn{2}{|c|}{ Total }} & 73 & 246 & 65 & 254 & 319 \\
\hline & & $22.9 \%$ & $77.1 \%$ & $20.4 \%$ & $79.6 \%$ & $100 \%$ \\
\hline
\end{tabular}

Seventy one (22.3\%) participants had discussed pubertal issues with the students and $113(35.4 \%)$ participants had discussed issue like HIV in their classrooms. Regarding issue of orientation of college students towards reproductive health through curriculum, $230(72.1 \%)$ teachers were in favour and $213(66.8 \%)$ were ready to advice students regarding contraceptives.

Two hundred twenty three $(69.9 \%)$ teachers ready for health training; while 271 (85\%) were in favour of induction of health cell in the college.
Knowledge about psychological problems was associated with adolescent health training. More than $40 \%$ participants who had undergone adolescent health training had good knowledge as compared to less than $30 \%$ participants who never had adolescent health training. However, this was not associated with gender, age, designation and experience. Knowledge about substance abuse was associated with designation; tutors and lecturers had better knowledge as compared to associate professors and professors. However, it was not associated with gender, age, experience or adolescent health training. (Table II) 
Table II: Association of knowledge about psychological problems and substance abuse with various sociodemographic variables.

\begin{tabular}{|c|c|c|c|c|c|c|}
\hline \multirow{3}{*}{ Variables } & & \multicolumn{4}{|c|}{ Knowledge about } & \multirow{4}{*}{$\begin{array}{c}\text { Total } \\
113\end{array}$} \\
\hline & & \multicolumn{2}{|c|}{ Psychological problems } & \multicolumn{2}{|c|}{ Substance abuse } & \\
\hline & & Poor & Good & Poor & Good & \\
\hline \multirow{5}{*}{ Gender } & \multirow{2}{*}{ Female } & 71 & 42 & 104 & 9 & \\
\hline & & $62.8 \%$ & $37.2 \%$ & $92 \%$ & $8 \%$ & $100 \%$ \\
\hline & \multirow{2}{*}{ Male } & 144 & 62 & 184 & 22 & 206 \\
\hline & & $69.9 \%$ & $30.1 \%$ & $89.3 \%$ & $10.7 \%$ & $100 \%$ \\
\hline & & \multicolumn{2}{|c|}{ Chi-square $=1.66, p=0.20$} & \multicolumn{2}{|c|}{ Chi-square $=0.613, p=0.43$} & \\
\hline \multirow{5}{*}{ Age } & \multirow{2}{*}{$\leq 40$} & 116 & 47 & 144 & 19 & 163 \\
\hline & & $71.2 \%$ & $28.8 \%$ & $88.3 \%$ & $11.7 \%$ & $100 \%$ \\
\hline & \multirow{2}{*}{$>40$} & 99 & 57 & 144 & 12 & 156 \\
\hline & & $63.5 \%$ & $36.5 \%$ & $92.3 \%$ & $7.7 \%$ & $100 \%$ \\
\hline & & Chi-squ & $=0.14$ & Chi-squ & $=0.23$ & \\
\hline \multirow{5}{*}{ Designation } & \multirow{2}{*}{$\begin{array}{l}\text { Tutor/ } \\
\text { Lecturer }\end{array}$} & 132 & 65 & 172 & 25 & 40 \\
\hline & & $67.0 \%$ & $33.0 \%$ & $87.3 \%$ & $12.7 \%$ & $100 \%$ \\
\hline & \multirow{2}{*}{$\begin{array}{l}\text { Associate } \\
\text { Professor/ } \\
\text { Professor }\end{array}$} & 83 & 39 & 116 & 6 & 157 \\
\hline & & $68.0 \%$ & $32.0 \%$ & $95.1 \%$ & $4.9 \%$ & $100 \%$ \\
\hline & & \multicolumn{2}{|c|}{ Chi-square $=0.36, p=0.85$} & \multicolumn{2}{|c|}{ Chi-square $=5.19, p=0.02$} & \\
\hline \multirow{5}{*}{ Experience } & \multirow{2}{*}{$\leq 5 \mathrm{yrs}$} & 56 & 22 & 68 & 10 & 78 \\
\hline & & $71.8 \%$ & $28.2 \%$ & $87.2 \%$ & $12.8 \%$ & $100 \%$ \\
\hline & \multirow{2}{*}{$>5$ yrs } & 159 & 82 & 220 & 21 & 241 \\
\hline & & $66 \%$ & $34 \%$ & $91.3 \%$ & $8.7 \%$ & $100 \%$ \\
\hline & & Chi-squ & $=0.34$ & Chi-squ & $=0.29$ & \\
\hline \multirow{5}{*}{$\begin{array}{l}\text { Adolescent } \\
\text { Health } \\
\text { training }\end{array}$} & \multirow{2}{*}{ No } & 162 & 67 & 210 & 19 & 229 \\
\hline & & $70.7 \%$ & $29.3 \%$ & $91.7 \%$ & $8.3 \%$ & $100 \%$ \\
\hline & \multirow{2}{*}{ Yes } & 53 & 37 & 78 & 12 & 90 \\
\hline & & $58.9 \%$ & $41.1 \%$ & $86.7 \%$ & $13.3 \%$ & $100 \%$ \\
\hline & & Chi-squ & $=0.04$ & Chi-squ & $=0.17$ & \\
\hline \multirow{2}{*}{\multicolumn{2}{|c|}{ Total }} & 215 & 104 & 288 & 31 & 319 \\
\hline & & $67.4 \%$ & $32.6 \%$ & $90.3 \%$ & $9.7 \%$ & $100 \%$ \\
\hline
\end{tabular}

Practices regarding psychological problems were associated with experience. Higher percentage of participants having experience less than five years had good practices regarding psychological problems as compared to those who have more experience.

However, it was not associated with gender, age, designation or adolescent health training. While practices regarding behavioural problems were associated with gender with more females exhibiting good practices as compared to males.

Similarly, practices about behavioural problems and substance abuse were associated with adolescent health training; higher percentage of participants with training had good practices as compared to others. (Table III) 
Table III: Association of practices regarding psychological problems, substance abuse and behavioural problems with various socio-demographic variables.

\begin{tabular}{|c|c|c|c|c|c|c|c|c|}
\hline \multirow{3}{*}{ Variables } & & \multicolumn{6}{|c|}{ Practices regarding } & \multirow{3}{*}{ Total } \\
\hline & & \multicolumn{2}{|c|}{$\begin{array}{l}\text { Psychological } \\
\text { problems }\end{array}$} & \multicolumn{2}{|c|}{$\begin{array}{l}\text { Substance } \\
\text { abuse }\end{array}$} & \multicolumn{2}{|c|}{$\begin{array}{l}\text { Behavioural prob- } \\
\text { lems }\end{array}$} & \\
\hline & & Poor & Good & Poor & Good & Poor & Good & \\
\hline \multirow{5}{*}{ Gender } & \multirow{2}{*}{ Female } & 104 & 9 & 110 & 3 & 89 & 24 & 113 \\
\hline & & $92 \%$ & $8 \%$ & $97.3 \%$ & $2.7 \%$ & $78.8 \%$ & $21.2 \%$ & $100 \%$ \\
\hline & \multirow{2}{*}{ Male } & 193 & 13 & 194 & 12 & 187 & 19 & 206 \\
\hline & & $93.7 \%$ & $6.3 \%$ & $94.2 \%$ & $5.8 \%$ & $90.8 \%$ & $9.2 \%$ & $100 \%$ \\
\hline & & \multicolumn{2}{|c|}{ Chi-square $=0.31, p=0.58$} & \multicolumn{2}{|c|}{$\begin{array}{l}\text { Chi-square }=1.64, \\
p=0.20\end{array}$} & \multicolumn{2}{|c|}{$\begin{array}{l}\text { Chi-square }=9.03, \\
p=0.003\end{array}$} & \\
\hline \multirow{5}{*}{ Age } & \multirow{2}{*}{$\leq 40$} & 153 & 10 & 157 & 6 & 138 & 25 & 163 \\
\hline & & $93.9 \%$ & $6.1 \%$ & $96.3 \%$ & $3.7 \%$ & $84.7 \%$ & $15.3 \%$ & $100 \%$ \\
\hline & \multirow{3}{*}{$>40$} & 144 & 12 & 147 & 9 & 138 & 18 & 156 \\
\hline & & $92.3 \%$ & $7.7 \%$ & $94.2 \%$ & $5.8 \%$ & $88.5 \%$ & $11.5 \%$ & $100 \%$ \\
\hline & & \multicolumn{2}{|c|}{ Chi-square $=0.30, p=0.58$} & \multicolumn{2}{|c|}{$\begin{array}{l}\text { Chi-square }=0.78 \\
p=0.38\end{array}$} & \multicolumn{2}{|c|}{$\begin{array}{l}\text { Chi-square }=0.99 \\
p=0.32\end{array}$} & \\
\hline \multirow{5}{*}{ Designation } & \multirow{2}{*}{$\begin{array}{l}\text { Tutor/ } \\
\text { Lecturer }\end{array}$} & 184 & 13 & 191 & 6 & 169 & 28 & 197 \\
\hline & & $93.4 \%$ & $6.6 \%$ & $97.0 \%$ & $3.0 \%$ & $85.8 \%$ & $14.2 \%$ & $100 \%$ \\
\hline & Associate & 113 & 9 & 113 & 9 & 107 & 15 & 122 \\
\hline & Professor & $92.6 \%$ & $7.4 \%$ & $92.6 \%$ & $7.4 \%$ & $87.7 \%$ & $12.3 \%$ & $100 \%$ \\
\hline & & \multicolumn{2}{|c|}{ Chi-square $=0.07, p=0.79$} & \multicolumn{2}{|c|}{$\begin{array}{l}\text { Chi-square }=3.15, \\
p=0.08\end{array}$} & \multicolumn{2}{|c|}{$\begin{array}{l}\text { Chi-square }=0.24, \\
p=0.63\end{array}$} & \\
\hline \multirow{5}{*}{ Experience } & \multirow{2}{*}{$\leq 5 \mathrm{yrs}$} & 68 & 10 & 72 & 6 & 63 & 15 & 78 \\
\hline & & $87.2 \%$ & $12.8 \%$ & $92.3 \%$ & $7.7 \%$ & $80.8 \%$ & $19.2 \%$ & $100 \%$ \\
\hline & \multirow{3}{*}{$>5$ yrs } & 229 & 12 & 232 & 9 & 213 & 28 & 241 \\
\hline & & $95 \%$ & $5 \%$ & $96.3 \%$ & $3.7 \%$ & $88.4 \%$ & $11.6 \%$ & $100 \%$ \\
\hline & & \multicolumn{2}{|c|}{ Chi-square $=5.64, p=0.018$} & \multicolumn{2}{|c|}{$\begin{array}{l}\text { Chi-square=2.06, } \\
p=0.15\end{array}$} & \multicolumn{2}{|c|}{$\begin{array}{l}\text { Chi-square }=2.93 \\
p=0.09\end{array}$} & \\
\hline \multirow{5}{*}{$\begin{array}{l}\text { Adolescent } \\
\text { Health } \\
\text { training }\end{array}$} & $\mathrm{N}$ & 213 & 16 & 226 & 3 & 214 & 15 & 229 \\
\hline & NO & $93.0 \%$ & $7.0 \%$ & $98.7 \%$ & $1.3 \%$ & $93.4 \%$ & $6.6 \%$ & $100 \%$ \\
\hline & Yec & 84 & 6 & 78 & 12 & 62 & 28 & 90 \\
\hline & res & $93.3 \%$ & $6.7 \%$ & $86.7 \%$ & $13.3 \%$ & $68.9 \%$ & $31.1 \%$ & $100 \%$ \\
\hline & & Chi-squ & $p=0.92$ & $\begin{array}{l}\text { Chi-squ } \\
p=0.00\end{array}$ & 20.8 & $\begin{array}{l}\text { Chi-sq } \\
p=0.00\end{array}$ & 33.4 & \\
\hline Tatal & & 297 & 22 & 304 & 15 & 276 & 43 & 319 \\
\hline rotal & & $93.1 \%$ & $6.9 \%$ & $95.3 \%$ & $4.7 \%$ & $86.5 \%$ & $13.5 \%$ & $100 \%$ \\
\hline
\end{tabular}

There was no statistical association between practices regarding psychological problems and substance abuse with their respective knowledge. Similarly, awareness regarding adolescent health problems was not associated with these practices.

Using SPSS 22, binary logistic regression models were made by Wald's backward method to find best predictors for attitude towards reproductive health and practices regarding psychological problems, substance abuse and behavioural problems.
Chance accuracy rate for all the models was greater than calculated accuracy rate. Experience was predictor for attitude towards reproductive health and practices regarding psychological problems and substance abuse; while age was a predictor for all the three practices. Knowledge regarding substance abuse was a predictor for its respective practices. (Table IV) 
Table IV: Binary logistic regression models-association of age, gender, experience, adolescent health training and respective knowledge with Attitude towards reproductive health and practices regarding Psychological problems, Substance abuse and Behavioural problems.

\begin{tabular}{|c|c|c|c|c|c|c|c|c|}
\hline \multirow{2}{*}{ Independent factor } & \multirow{2}{*}{ B } & \multirow{2}{*}{ S.E. } & \multirow{2}{*}{ Wald } & \multirow{2}{*}{ d.f } & \multirow{2}{*}{$P$ value } & \multirow{2}{*}{ OR } & \multicolumn{2}{|c|}{$95 \% \mathrm{Cl}$ for $\mathrm{OR}$} \\
\hline & & & & & & & Lower & Upper \\
\hline \multicolumn{9}{|c|}{ Model 1: Dependent factor $=$ Attitude towards reproductive health; model accuracy $=79.6 \%$. } \\
\hline Gender & -.439 & .303 & 2.096 & 1 & .148 & .645 & .356 & 1.168 \\
\hline Experience & -.032 & .015 & 4.742 & 1 & .029 & .969 & .941 & .997 \\
\hline Constant & 2.03 & .333 & 37.142 & 1 & .000 & 7.633 & & \\
\hline \multicolumn{9}{|c|}{ Model 2: Dependent factor = Practices regarding psychological problems; model accuracy $=99 \%$. } \\
\hline Age & .383 & .174 & 4.841 & 1 & .028 & 1.467 & 1.043 & 2.064 \\
\hline Experience & -.288 & .147 & 3.844 & 1 & .050 & .749 & .562 & 1.000 \\
\hline Constant & -16.91 & 6.347 & 7.099 & 1 & .008 & .000 & & \\
\hline \multicolumn{9}{|c|}{ Model 3: Dependent factor $=$ Practices regarding substance abuse; model accuracy $=98.1 \%$. } \\
\hline Age & .268 & .120 & 5.022 & 1 & .025 & 1.308 & 1.034 & 1.654 \\
\hline Experience & -.322 & .137 & 5.522 & 1 & .019 & .725 & .554 & .948 \\
\hline $\begin{array}{l}\text { Knowledge regarding } \\
\text { substance abuse }\end{array}$ & 2.682 & .967 & 7.695 & 1 & .006 & 14.610 & 2.197 & 97.178 \\
\hline Constant & -13.76 & 4.151 & 10.988 & 1 & .001 & .000 & & \\
\hline \multicolumn{9}{|c|}{ Model 4: Dependent factor = Practices regarding behavioural problems; model accuracy $=90.8 \%$. } \\
\hline Age & -.107 & .027 & 15.375 & 1 & .000 & .899 & .852 & .948 \\
\hline Constant & 1.53 & .904 & 2.866 & 1 & .090 & 4.620 & & \\
\hline
\end{tabular}

By applying Spearman's rank correlation; practices regarding psychological problems and behavioural problems had a positive moderate correlation $(p<$ 0.05) with attitude towards reproductive health. Practices regarding psychological problems and substance abuse also had a positive moderate correlation with practices regarding behavioural problems $(p<0.05)$.

\section{DISCUSSION}

We observed that majority of teachers had awareness regarding adolescent health problems and a positive attitude towards reproductive health. Briggs LA observed that only $48.3 \%$ teachers from Nigeria advocates sex education program in the curriculum. ${ }^{15}$ In a study conducted in Nigeria by Orji and Esimai, ${ }^{16}$ it was observed that $90 \%$ teachers were in favour of inclusion of sex education program in curriculum. Aniebue PN observed that 94\% of teachers from Enugu were positive towards such inclusion. ${ }^{17}$ In the current study, $72 \%$ of teachers were in favour of orientation of students to reproductive health education. This proportion although lower that observed by Orji et al. and Aniebue, but still very high as compared to that observed by Briggs. The socio-cultural differences may be responsible for wide range of differences in observations regarding the issue.
Briggs observed that only $20.8 \%$ teachers were in favour of advising students about contraceptives. ${ }^{15}$ While we observed that, $66.8 \%$ teachers were in favour. The differences can be due cultural variation as well as higher age group of students tutored by the teachers in our study.

Nair et al, observed that $40.9 \%$ school teachers from Thiruvanthapuram had actually discussed aspects growth with adolescents. ${ }^{18}$ While we observed that only $22.5 \%$ teachers had such discussion, however our study participants were college teachers. The students being already in the late adolescents may not seek help regarding pubertal issue from teachers.

The communication with students was lacking, with less than quarter of teachers ever been approached by students regarding health queries, while only $36 \%$ of them had discussed issues like HIV and STD with students.

Most of the teachers had poor knowledge regarding the mental health and substance abuse. Age, gender and experience had no effect on the knowledge. Teachers who had some training previously were better; but overall knowledge level was poor. Training received by the teachers was not compulsory and refresher training was never 
conducted. There is no system in India to actually test the knowledge of teachers regarding such subjects.

The practices of the majority of teachers regarding mental health problems, substance abuse and behavioural problems were poor. Adolescent health training helped in development of better practices regarding adolescent health, among the teachers. Younger teachers fared better in practices.

It seems that teachers who have been through this phase recently were more sympathetic and involved. Female teachers had better practices regarding behavioural problems; which may be due to higher acceptances of males towards such behaviour.

During the course of data collection, some of the teachers had discussed their own habit of substance abuse. Many of such teachers were unable to advice the students against substance abuse due to feeling of guilt or not wanting to sound hypocritical.

\section{Limitations of the study}

The study is based on self-addressed questionnaires, hence reporting bias cannot be completely eliminated. There may be over estimation of positive attitude and good practices. There is no mechanism to confirm the findings.

\section{CONCLUSION}

Majority of teacher had good attitude and awareness regarding various adolescent issues. But the knowledge and practices regarding such issue were underwhelming. Communication of teachers with students was poor. Health training had positive impact on the practices of some adolescent issues; however total teachers receiving the training was low. Similar overall practices were better in younger staff.

It is recommended that, all the teachers must undergo training regarding adolescent health; with periodic refresher courses or lectures. They should be taught to identify symptoms of psychological health problems and substance abuse. Efforts should be made to develop positive attitude and sense of responsibility towards adolescent health in the teachers. They should be encouraged to discuss issues like contraception, STD/HIV with their students.

Active efforts to bridge the communication gap between teachers and students should be taken. Anonymous feedback from students should be sought to keep vigilance over efforts of teachers to communicate with students. Teachers with problem of substance abuse should be identified and encouraged to undergo de-addiction programme, to set a proper example among students.

\section{Conflicts of Interest}

None to declare.

\section{Authors' contribution}

All authors contributed to conception and design and drafting the manuscript. VBW and MSC contributed to development of research tool and procedure. VBW and ADG acquired and analyzed the data. VBW, $A D G$ and GBD contributed to interpretation of data. All authors have read and approved the final manuscript.

\section{Acknowledgement}

We would like to thank all the institutes and study participants for giving their valuable time and cooperation for this research.

\section{REFERENCES}

1. World Health Organization. The second decade: improving adolescent health and development. Geneva: World Health Organization, 2001.

2. Sawyer SM, Afifi RA, Bearinger LH, et al. Adolescence: a foundation for future health. Lancet 2012;379:1630-40.

3. Patton GC, Viner RM. Pubertal transitions in health. Lancet 2007;369:1130-9.

4. United Nations, Department of Economic and Social Affairs. World population prospects: the 2010 revision. New York, NY: United Nations 2011.

5. United Nations Children's Fund. Adolescence: An age of Opportunity. New York, NY. United Nations Children's Fund 2011.

6. Tonin V. Young people seeking mental-health care. Lancet 2007;369:1239-40.

7. Gulliver A, Griffiths KM, Christensen $\mathrm{H}$. Perceived barriers and facilitators to mental health help-seeking in young people: a systematic review. BMC Psychiatry 2010;10:113.

8. Kishore J, Gupta A, Jiloha RC, Bantman P. Myths, beliefs and perceptions

about mental disorders and healthseeking behavior in Delhi, India. Indian J Psychiatry. 2011;53:324-9.

9. Bruwer B, Sorsdahl K, Harrison J, Stein DJ, Williams D, Seedat S. Barriers to mental health care and predictors of treatment dropout in the South African Stress and Health Study. Psychiatr Serv. 2011;62:77481.

10. Devgan S, Padda AS, Deepti SS, Kaur J, Ahlluwalia SK. Effectiveness of health education among female teachers of senior secondary regarding problems in adolescence. Indian Journal of Community Health 2012; 24:107-12.

11. American Psychiatric Association. Diagnostic and statistical manual of mental disorders. $4^{\text {th }}$ edn. Text rev. Washington, DC: American 
Psychiatric Association, 2000.

12. Shastri PC, Shastri JP. Psychiatric disorders: Interview, assessment and diagnosis. In: Bhave SY, editor. Bhave's Textbook of Adolescent Medicine. New Delhi: Jaypee Brothers Medical Publishers (P) Ltd; 2006. p.749-52.

13. Greydanus DE, Yadav S. Substance abuse disorders. In: Bhave SY, editor. Bhave's Textbook of Adolescent Medicine. New Delhi: Jaypee Brothers Medical Publishers (P) Ltd; 2006. p.800-5.

14. Jain T, Bisht. Impact of Violence. In: Bhave SY, editor. Bhave's Textbook of Adolescent Medicine. New Delhi: Jaypee Brothers Medical Publishers (P) Ltd; 2006. p.907-11.

15. Briggs LA. Secondary school teachers opinion about contraceptive practice and pregnancy among school girls in Port Harcourt, Nigeria: implications for family planning programmes. Malays J Reprod Health 1994;12:1-9.

16. Orji EO, Esimai OA . Introduction of sex education into Nigerian schools: the parents', teachers' and students' perspectives. J Obstet Gynaecol. 2003;23:185-8.

17. Aniebue PN. Knowledge and attitude of secondary school teachers in Enugu to school based sex education. Niger J Clin Pract. 2007;10:304-8.

18. Nair MK, Leena ML, Paul MK, et al. Attitude of Parents and Teachers towards Adolescent Reproductive and Sexual Health Education. Indian J Pediatr 2012;79:60-3. 